\title{
Preparation and Evaluation of Doxorubicin-Loaded Micelles Based on Glycyrrhetinic Acid Modified Gelatin Conjugates for Targeting Hepatocellular Carcinoma
}

\author{
Dun Fan, ${ }^{1,2}$ Jingmou Yu $\mathbb{D}^{1,3}$ Ruiqiao Yan, ${ }^{4}$ Xiao Xu, ${ }^{4}$ Yunfei Wang, ${ }^{4}$ Xin Xie, \\ Chaolian Liu, ${ }^{1}$ Yonghua Liu, ${ }^{4}$ and Hao Huang $\mathbb{D}^{2}$ \\ ${ }^{1}$ School of Pharmacy and Life Sciences, Jiujiang University, Jiujiang 332000, China \\ ${ }^{2}$ School of Chemical and Biological Engineering, Yichun University, Yichun 336000, China \\ ${ }^{3}$ Key Laboratory of Jiangxi Province for Systems Biomedicine, Jiujiang University, Jiujiang 332000, China \\ ${ }^{4}$ Affiliated Hospital of Jiujiang University, Jiujiang 332000, China \\ Correspondence should be addressed to Jingmou Yu; yjm1016@hotmail.com and Hao Huang; jxychh2008@gmail.com
}

Received 17 August 2017; Accepted 22 October 2017; Published 1 January 2018

Academic Editor: Abdelwahab Omri

Copyright (C) 2018 Dun Fan et al. This is an open access article distributed under the Creative Commons Attribution License, which permits unrestricted use, distribution, and reproduction in any medium, provided the original work is properly cited.

\begin{abstract}
Hepatocellular carcinoma (HCC) is one of the most prevalent fatal diseases and the incidence of HCC is increasing worldwide. Polymeric micelles with targeting groups have drawn great attention as carriers for drug delivery in HCC therapy. Herein, novel glycyrrhetinic acid modified gelatin (GA-GEL) conjugates with three substitution degrees were synthesized and characterized. Doxorubicin (DOX) was applied as a model drug. DOX-loaded GA-GEL (DOX/GA-GEL) micelles were prepared by an emulsionsolvent evaporation method. The mean diameters of DOX/GA-GEL micelles were in the range of 195-235 nm. The encapsulation efficiency of DOX/GA-GEL micelles was $63.6 \%-96.2 \%$, and the loading content was $8.3 \%-12.5 \%$. Drug release from DOX-loaded micelles exhibited a biphasic manner in phosphate buffer solution (PBS) at pH 7.4. DOX/GA-GEL could be efficiently accumulated into human liver cancer HepG2 cells. The $\mathrm{IC}_{50}$ values of DOX/GA-GEL-2 and DOX·HCl in HepG2 cells were 0.33 and $0.66 \mu \mathrm{g} / \mathrm{mL}$, respectively. In vivo imaging analysis demonstrated that the fluorescence signals of DiR-labeled GA-GEL-2 micelles were mainly distributed in liver and H22 orthotopic tumor, indicating that GA-GEL had the liver-targeting activity. Compared to DOX.HCl, DOX/GA-GEL-2 exhibited better antitumor activity in H22 orthotopic mice. Therefore, these results indicated that GA-GEL could be used as carrier of hydrophobic drug for targeting HCC.
\end{abstract}

\section{Introduction}

Nowadays, HCC is one of the most severe diseases in the world, and terribly threaten human health because of its high morbidity and mortality [1]. As we know, drug therapy was still indispensable to HCC treatment. Traditional chemotherapeutic drug, however, remained to have several problems including poor selectivity and solubility as well as serious side effects. Then it could decrease antitumor drug accumulation in targeting tissue and weaken the antitumor activity [2]. In recent years, nanoscaled drug delivery system, such as polymeric micelles, had been concerned by many researchers, owing to its perfect targeting, good solubility, altering tissue distribution, and controlled release characteristics [3]. Amphiphilic copolymers can form nanoscaled micelles with core-shell structure in aqueous media via selfassembly $[4,5]$. The hydrophobic core serves as a reservoir for incorporating hydrophobic drugs [6-9]. The hydrophilic shell could reduce the interaction with plasma proteins and prolong the blood circulation time. Self-assembled micelles can reduce toxic side effects and improve therapeutic effects. Over the past decades, much attention has been paid to prepare biodegradable polymeric amphiphiles based on natural materials such as chitosan and gelatin.

Gelatin (GEL), a kind of proteins purified from skin and bone of animals, has been recognized as safe material. It could 
be dissolved in aqueous media. Moreover, gelatin is the substrate of matrix metalloproteinase-2 (MMP-2). And gelatinbased nanoparticles could be degraded by MMP-2, and drug encapsulated could be quickly released in the tumor sites [10]. As previously described, gelatin-based micelles had the advantages of biocompatible and biodegradable properties without obvious toxicity and immunogenicity [11, 12].

Polymeric amphiphiles with targeting groups have been extensively studied and employed as effective carriers of drug and gene. It has been testified that liver-targeting functional ligands could interact with the corresponding receptors on hepatocyte surface [13]. And the liver-targeting activity of antitumor drug was improved as the ligand was introduced into nanoscaled drug carrier, such as folic acid, lactose acid, and glycyrrhetinic acid [14-16]. Glycyrrhetinic acid (GA) is a bioactive compound, which is extracted from root and rhizome of Chinese traditional herb licorice. GA could specially bind with GA receptor, and GA-modified micelles could be effectively transported into hepatic cells by endocytosis $[17,18]$.

Up to now, many studies on GA-modified micelles as drug carriers targeting to HCC had been carried out $[19,20]$. Chen et al. had constructed liver-targeting and redox-responsive micelles by GA coupling with poly(ethylene glycol)-disulfide linkage-poly(lactic-co-glycolic acid). Tanshinone IIA (TAN IIA) was encapsulated, and drug-loaded micelles resulted in an increased accumulation of TAN IIA in the liver. With the synergistic effects of HCC-targeting and controlled drug release, TAN IIA-loaded micelles markedly inhibited tumor growth and increased survival period in HCC-xenograft mice model [17]. In addition, the uptake of DOX-loaded GA-modified sulfated chitosan by HepG2 cells was about 4.48-folds that of DOX-loaded stearic acid modified sulfated chitosan [21]. Further, GA-modified polypropylenimine dendrimers with various substitution ratio could effectively transport DNA into HepG2 cells [22].

In the present study, the objective is to construct novel liver-targeting micelles based on GA-modified GEL (GA-GEL) copolymers. GA-GEL conjugates with different degree of substitution were synthesized and characterized. Their physicochemical properties were investigated. DOX as a model antitumor drug was encapsulated into GA-GEL micelles. In vitro release behaviors of DOX-loaded micelles were performed in PBS. The cellular uptake of DOX/GA-GEL micelles was studied, and in vitro cytotoxicity was conducted in HepG 2 cells. Moreover, in vivo imaging analysis, antitumor activities, and safety evaluation of drug-loaded micelles were investigated in detail.

\section{Materials and Methods}

2.1. Materials. GA, 1-ethyl-3-(3-dimethylaminopropyl) carbodiimide hydrochloride (EDC), and $N$-hydroxysuccinimide (NHS) were purchased from Aladdin Industrial Corporation (Shanghai, China). Gelatin (type A) and 2,4,6-trinitrobenzenesulfonic acid (TNBS) were obtained from SigmaAldrich (St. Louis, OM, USA). Doxorubicin hydrochloride (DOX. $\mathrm{HCl}$ ) was from Beijing Huafeng United Technology
Co. Ltd. (Beijing, China). Hoechst 33258 was purchased from Beyotime Institute of Biotechnology (Haimen, China). 3-(4,5-dimethyl-thiozol-2-yl)-2,5-diphenyl-tetrazolium bromide (MTT) was obtained from Sigma-Aldrich (St. Louis, OM, USA). RPMI1640 medium and trypsin-EDTA were purchased from Jinuo Biotechnology Company (Hangzhou, China). Fetal bovine serum (FBS) was provided by Sijiqing Biological Co. Ltd. (Hangzhou, China). Matrigel Matrix was obtained from Becton, Dickinson, and Company (Franklin Lake, USA). DiR iodide [1,1-dioctadecyl-3,3,3,3tetramethylindotricarbocyanine iodide] was purchased from AAT Bioquest (Sunnyvale, CA, USA). All other chemical reagents were of analytical grade.

HepG2 cells were provided by the Institute of Biochemistry and Cell Biology Chinese Academy Sciences (Shanghai, China). H22 cells were obtained from China Center for Type Culture Collection (Wuhan, China). Male Kunming mice $(20 \pm 2 \mathrm{~g}$ ) were from Hunan SLAC Jingda Laboratory Animal Co. Ltd. (Changsha, China). All animal experiments were complied with the international regulations for animal experimentation.

2.2. Synthesis of GA-GEL Conjugates. GA-GEL copolymers were synthesized by EDC reaction method. Specifically, GEL (1.0 g) was dissolved in $100 \mathrm{~mL}$ of dimethyl sulfoxide (DMSO) and water $(7: 3, \mathrm{v}: \mathrm{v})$ and was stirred at $60^{\circ} \mathrm{C}$ for $30 \mathrm{~min}$. GA $(0.05 \mathrm{~g})$ dissolved in DMSO $(2.5 \mathrm{~mL})$ was dropwise added to the above solution. Then EDC $(0.03 \mathrm{~g})$ and NHS $(0.018 \mathrm{~g})$ were added. The reaction mixture was carried out at $35^{\circ} \mathrm{C}$ for $24 \mathrm{~h}$. Further, the mixed solution was dialyzed against deionized water (MWC: $14 \mathrm{kDa}$ ) for $48 \mathrm{~h}$ and lyophilized. Finally, GA-GEL-1 conjugate was obtained. The same method was used to further synthesize two kinds of copolymers. Then GA-GEL conjugates with various feed mass ratios of GA to gelatin (1:10 and 1:5) were denoted as GA-GEL-2 and GAGEL-3.

\subsection{Preparation of DOX/GA-GEL and DiR-Labeled GA-} GEL Micelles. DOX-loaded micelles were prepared by an emulsion-solvent evaporation method [23]. DOX. $\mathrm{HCl}$ was dissolved in dichloromethane with three equivalent molar ratios of triethylamine to eliminate hydrochloride acid. And the solution was stirred $12 \mathrm{~h}$ under the dark condition. DOX $(15 \mathrm{mg})$ in dichloromethane $(5 \mathrm{~mL})$ was dropwise added to blank micelles $(1 \mathrm{mg} / \mathrm{mL}, 100 \mathrm{~mL})$ under high speed stirring in ice bath. The mixture solution was magnetically stirred for $3 \mathrm{~h}$. Then dichloromethane was evaporated by using rotary evaporator under reduced pressure. DOX-loaded micelles were filtered through $0.8 \mu \mathrm{m}$ membrane to remove unloaded DOX. After lyophilization, DOX-loaded GA-GEL micelles were obtained.

Similar method was performed to prepare the DiRlabeled GA-GEL-2 micelles for in vivo imaging analysis. Except that DOX (15 mg) dichloromethane solution was replaced by $\mathrm{DiR}(2 \mathrm{mg}$ ) dichloromethane solution, the following procedure was exactly the same as described above. 


\subsection{Characterization of Blank and DOX-Loaded Micelles}

2.4.1. Characterization of GA-GEL Conjugates. GA-GEL copolymers were dissolved in the mixture of $\mathrm{D}_{2} \mathrm{O}$ and $d$-DMSO $(1: 3, \mathrm{v}: \mathrm{v})$. The structures were confirmed by ${ }^{1} \mathrm{H}$ nuclear magnetic resonance $\left({ }^{1} \mathrm{H}\right.$ NMR) spectrometer (AVANCE DMX 500, Bruker, Germany). The substitution degrees of amino groups were determined by TNBS method [24, 25]. Briefly, $2 \mathrm{mg} / \mathrm{mL}$ of gelatin solution was prepared. A serial of stock solution was taken out, and deionized water was added to a final volume of $2 \mathrm{~mL}$. Then $2 \mathrm{~mL}$ of $4 \%$ sodium hydrogen carbonate and $2 \mathrm{~mL}$ of $0.1 \%$ TNBS were added. The above solution was incubated in $37^{\circ} \mathrm{C}$ for $2 \mathrm{~h}$. The absorbance of each sample was detected by UV/VIS spectrophotometer (1700 DB, Shimadzu, Kyoto, Japan) in $350 \mathrm{~nm}$. The calibration curve was obtained according to the measured data. Three kinds of GA-GEL micelles $(2 \mathrm{mg} / \mathrm{mL})$ were carried out by the above method. And their substitution degrees were determined.

2.4.2. Critical Micelle Concentration (CMC). CMC was detected by using pyrene as a probe with fluorescence spectroscopy [26]. Three kinds of blank conjugates were dissolved by deionized water. Pyrene in acetone $(1 \mathrm{~mL})$ was added into flasks, and acetone was evaporated in $50^{\circ} \mathrm{C}$ water bath. Then $10 \mathrm{~mL}$ of various concentration micelles were added into each flask and heated at $50^{\circ} \mathrm{C}$ for $10 \mathrm{~h}$ to equilibrate pyrene and micelles. The solution remained to cool for $8 \mathrm{~h}$ at room temperature. The final concentration of pyrene was $6.0 \times 10^{-7} \mathrm{M}$. The fluorescence spectra were scanned by fluorescence spectrophotometer (Perkin-Elmer LS55, Perkin-Elmer Ltd., Llantrisant, UK). The slit width was $10 \mathrm{~nm}$. The emission and excitation wavelengths were 339 and $390 \mathrm{~nm}$, respectively.

2.4.3. Particle Size of Blank and Drug-Loaded Micelles. $10 \mathrm{mg}$ of blank or drug-loaded micelles was dissolved in $10 \mathrm{~mL}$ deionized water. The mean diameters were determined by dynamic light scattering (DLS) using a Zetasizer (90Plus, Brookhaven Instruments Corp., New York, NY, USA). Morphological observation of GA-GEL-2 and DOX/GA-GEL2 micelles was done by transmission electron microscopy (TEM, JEM-1230, Jeol, Tokyo, Japan). The samples were prepared by deionized water and placed on copper grids. Then copper grids were negative stained by $1 \%(\mathrm{w} / \mathrm{v})$ phosphotungstic acid and were air-dried at room temperature before observation [27].

2.4.4. Loading Content (LC) and Encapsulation Efficiency (EE). DOX-loaded micelles were dissolved in deionized water, and DOX was extracted from the micelles by adding DMSO. LC and EE were defined by UV spectrophotometric analysis at $481 \mathrm{~nm}$. LC and EE were calculated by the following equations:

$$
\begin{aligned}
& \mathrm{LC}(\%)=\frac{\text { Weight of DOX in micelles }}{\text { Weight of DOX }- \text { loaded micelles }} \times 100 \%, \\
& \mathrm{EE}(\%)=\frac{\text { Weight of DOX in micelles }}{\text { Weight of DOX in feed }} \times 100 \% .
\end{aligned}
$$

2.5. In Vitro Drug Release. DOX release from DOX-loaded micelles was performed by dialysis method in PBS at $\mathrm{pH}$ 7.4 [28]. Specifically, $1 \mathrm{~mL}$ of the micelles added in dialysis bag (MWCO: $14 \mathrm{kDa}$ ) was immersed in $20 \mathrm{~mL}$ release media and kept at $37^{\circ} \mathrm{C}$ and $160 \mathrm{rpm}$ in an air-bath-shaker. $5 \mathrm{~mL}$ of sample solution outside the dialysis bag was taken out and supplemented with equal volume of fresh release media at appropriate intervals. The concentration of DOX was detected by fluorescence spectrophotometer (Perkin-Elmer LS55, Perkin-Elmer Ltd., Llantrisant, UK). The excitation and emission wavelengths were 470 and $585 \mathrm{~nm}$, respectively. Each sample was investigated in triplicate.

2.6. In Vitro Cellular Uptake. Confocal laser scanning microscope (CLSM) was employed to study in vitro cellular uptake [29]. HepG2 cells were incubated at $2 \times 10^{5}$ per well in 6 -well plates (Costar, Corning, NY, USA) for $24 \mathrm{~h}$. After the medium was removed, DOX.HCl or DOX-loaded GA-GEL micelles (equivalent DOX concentration: $5 \mu \mathrm{g} / \mathrm{mL}$ ) were added. The cells were cultured for 2 or $6 \mathrm{~h}$. Then the cells were washed twice with PBS ( $\mathrm{pH} 7.4$ ) and fixed in 4\% paraformaldehyde solution for $30 \mathrm{~min}$. For nuclei staining, the cells were treated with Hoechst $33258(5 \mathrm{mg} / \mathrm{mL})$ for $30 \mathrm{~min}$. HepG2 cells were washed with PBS and observed by a Zeiss LSM-510 confocal microscope (Carl Zeiss LSM-510, Germany).

Especially, in order to further investigate liver-targeting activity of DOX/GA-GEL-2 micelles, free GA $(12 \mu \mathrm{g} / \mathrm{mL})$ was preincubated with HepG 2 cells for $2 \mathrm{~h}$. Then DOX/GA-GEL2 micelles were added and cultured for $6 \mathrm{~h}$, and the following process was in accordance with the above state.

2.7. In Vitro Cytotoxicity. The cytotoxicity of blank or DOXloaded micelles was evaluated by using MTT assay [18]. HepG2 cells were seeded into 96-well plates (Costar, Corning, NY, USA) and incubated at a density of $1 \times 10^{4}$ cells per well for $24 \mathrm{~h}$. After the cultured medium was removed, various concentrations of DOX $\cdot \mathrm{HCl}$, blank, or DOX-loaded micelles were added. Then the media were discarded. $30 \mu \mathrm{L}$ of MTT solution was added and carried out for $4 \mathrm{~h}$. After MTT solution was aspirated, $200 \mu \mathrm{L}$ DMSO was used to solubilize formazan crystals in live cells. The cell viability was determined by detecting absorbance at $490 \mathrm{~nm}$ with a microplate reader (Thermo Scientific Multiskan MK3, Hudsan, USA).

2.8. H22 Orthotopic Xenograft Model. H22 orthotopic xenograft model was established as previously reported [30]. H22 cells cultured in the logarithmic phase were harvested by centrifugation. The cells were suspended with culture media at a density of $5 \times 10^{7} / \mathrm{mL}$, and $0.2 \mathrm{~mL}$ of matrix gel was added in $1 \mathrm{~mL}$ cell suspension. The cell suspension was kept on ice water. The laparotomy was performed to expose the left liver lobe where H22 cell suspension was injected with a $1 \mathrm{~mL}$ injection syringe. The depth of each injection into the lobe was about $2-3 \mathrm{~mm}$, and the injection volume was $0.05 \mathrm{~mL}$. A "white dot" at the injection site on the lobe appeared after successful injection. 


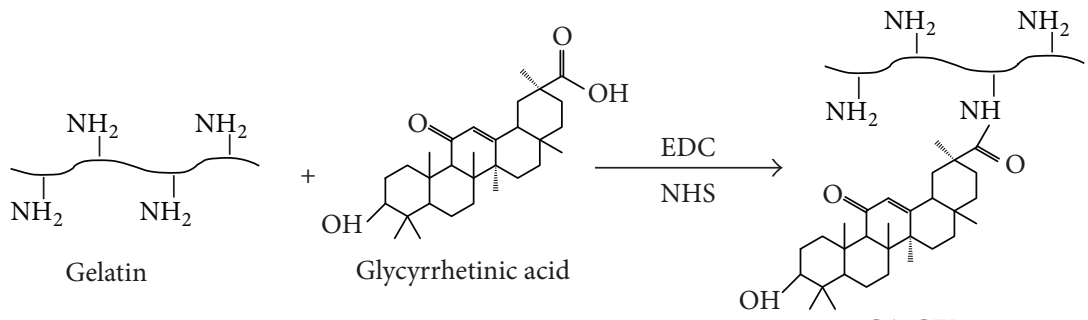

GA-GEL

FIgURE 1: Synthetic scheme of GA-GEL conjugate.

TABLE 1: Physicochemical characteristics of blank and DOX-drug micelles.

\begin{tabular}{|c|c|c|c|c|c|c|}
\hline Samples & $\operatorname{Size}^{\mathrm{a}}(\mathrm{nm})$ & LC $(\%)^{b}$ & $\mathrm{EE}(\%)^{\mathrm{c}}$ & Zeta potential $(\mathrm{mV})$ & $\mathrm{PI}^{\mathrm{d}}$ & DS (\%) ${ }^{\mathrm{e}}$ \\
\hline GA-GEL-1 & $119 \pm 18.5$ & - & - & $14.2 \pm 0.8$ & $0.321 \pm 0.008$ & 18.3 \\
\hline GA-GEL-2 & $121 \pm 22.4$ & - & - & $13.4 \pm 0.7$ & $0.171 \pm 0.008$ & 27.5 \\
\hline GA-GEL-3 & $134 \pm 16.3$ & - & - & $15.1 \pm 0.6$ & $0.151 \pm 0.021$ & 33.8 \\
\hline DOX/GA-GEL-1 & $195 \pm 21.5$ & $10.9 \pm 1.2$ & $83.8 \pm 9.3$ & $14.4 \pm 0.9$ & $0.198 \pm 0.003$ & - \\
\hline DOX/GA-GEL-2 & $210 \pm 19.4$ & $12.5 \pm 1.5$ & $96.2 \pm 11.6$ & $14.9 \pm 0.5$ & $0.286 \pm 0.025$ & - \\
\hline DOX/GA-GEL-3 & $235 \pm 25.8$ & $8.3 \pm 1.3$ & $63.6 \pm 10.7$ & $13.8 \pm 0.6$ & $0.250 \pm 0.006$ & - \\
\hline
\end{tabular}

${ }^{a}$ Measured by dynamic light scattering. ${ }^{b}$ Loading content. ${ }^{c}$ Encapsulation efficiency. ${ }^{\mathrm{d}}$ Polydispersity index. ${ }^{\mathrm{e}}$ Degree of substitution.

2.9. In Vivo Imaging Analysis. In vivo real-time fluorescence imaging analysis was used to evaluate the effect of tissue distribution and accumulation ability of GA-GEL-2 micelles in orthotopic $\mathrm{H} 22$ tumor-bearing mice [31]. As H22 cells were inoculated for 10 days, DiR-loaded GA-GEL-2 micelles were injected via tail vein at a DiR dose of $200 \mu \mathrm{g} / \mathrm{kg}$. The imaging was performed at $1,4,8,12$, and $24 \mathrm{~h}$ after injection by in vivo imaging system (DXS4000PRO, Kodak, USA). In order to represent the liver-targeting potential of GA-GEL-2 micelles, mice were humanely sacrificed at 1,12 , and $24 \mathrm{~h}$. Then the tumors and key organs (including heart, liver, spleen, lung, and kidney) were extracted, and the fluorescence intensity was determined with the same system as described above. The excitation and emission wavelengths were 748 and $780 \mathrm{~nm}$, respectively.

2.10. In Vivo Antitumor Efficacy and Safety Evaluation. In vivo antitumor efficacy and safety evaluation were conducted in $\mathrm{H} 22$ orthotopic xenograft mice [32]. After the inoculation was done on day $8, \mathrm{H} 22$ orthotopic xenograft mice were randomly divided into 4 groups $(n=6)$. Mice were treated with $5 \%$ glucose injection, DOX $\cdot \mathrm{HCl}(2.5 \mathrm{mg} / \mathrm{kg}), \mathrm{DOX} / \mathrm{GA}-$ GEL-2 (2.5 mg/kg on DOX basis), and GA-GEL-2 (20 mg/kg). Administration was performed for 4 times at a frequency of every 2 days. The body weight, viability, mental status, and adverse reactions of each mouse were investigated and recorded every day. On the 10th day, all mice were sacrificed by cervical dislocation. The tumors and organs were removed and fixed with $10 \%$ formaldehyde. The tumor weight was quantified by electronic balance, and tumor inhibitory rate (TIR) was calculated by the following formula:

$$
\operatorname{TIR}(\%)=\frac{W_{G}-W_{X}}{W_{G}} \times 100 \%,
$$

where $W_{G}$ represented the tumor weight of 5\% glucose group and $W_{X}$ stood for that of DOX.HCl or DOX/GA-GEL-2 group.

The organs and tumors were excised to make hematoxylin and eosin (H\&E) staining sections and visualized under a microscope (Leica DMI 4000B, Germany).

2.11. Statistical Analysis. All experimental data were given as mean \pm SD. Statistical significance was tested by two-tailed Student's $t$-test. The differences were judged to be significant at $P<0.05$.

\section{Results and Discussion}

3.1. Synthesis and Characterization of GA-GEL Conjugates. The synthesis procedure of GA-GEL was illustrated in Figure 1 . The copolymers were synthesized by the formation of amide bonds between amino groups of gelatin and carboxyl groups of GA. The structure of polymeric conjugates was confirmed by ${ }^{1} \mathrm{H}$ NMR spectra. As shown in Figure 2, compared with gelatin, new proton peaks of GA-GEL-1, GAGEL-2, and GA-GEL-3 were mainly observed in 0.6-1.0, 2.4, and $2.8 \mathrm{ppm}$. These peaks were assigned to GA molecules. As the feed mass ratio of GA increased, theses proton peaks appearing in three kinds of copolymers were obviously increased. The phenomenon is similar to the report described by Shi et al. [33]. TNBS method was used to study the substitution degrees of amino groups. Substitution degrees of GA-GEL-1, GA-GEL-2, and GA-GEL-3 were 18.3\%, 27.5\%, and $33.8 \%$, respectively (Table 1 ). The substitution degrees were increased as the feed mass ratio of GA was increased. These results were in accordance with ${ }^{1} \mathrm{H}$ NMR spectra. These results indicated that GA-GEL conjugates were successfully synthesized. 


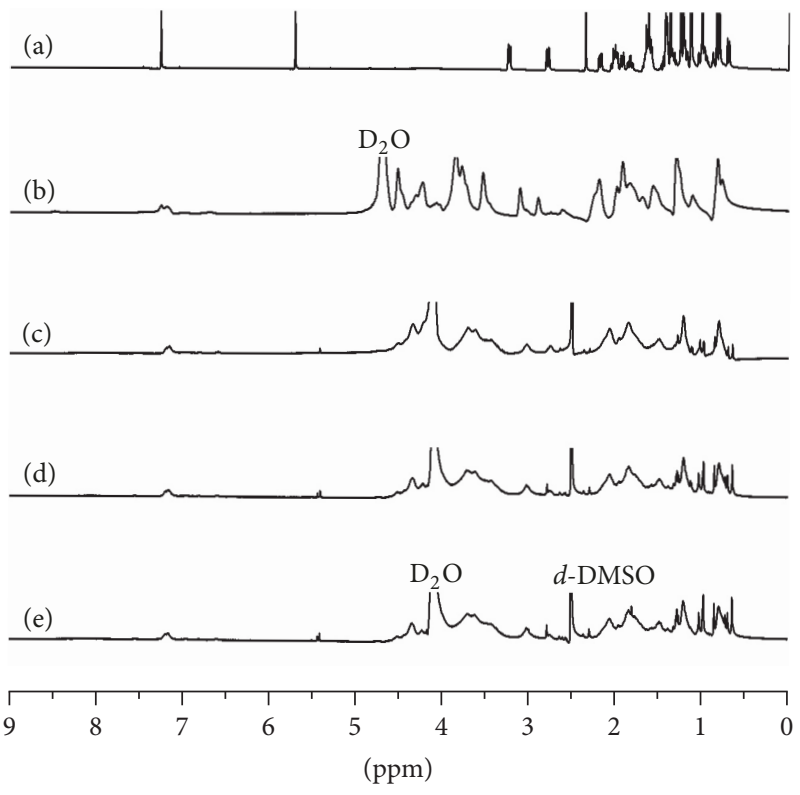

Figure 2: ${ }^{1} \mathrm{H}$ nuclear magnetic spectra of (a) GA, (b) gelatin, (c) GA-GEL-1, (d) GA-GEL-2, and (e) GA-GEL-3.

The CMC of GA-GEL conjugate was determined by fluorescence spectra using pyrene as a hydrophobic fluorescence probe [8]. CMC was assumed to be observed as there was a sharp increase in the ratio of the fluorescence intensities $\left(I_{338} / I_{333}\right)$. Figure 3 showed the intensity ratio of $I_{338} / I_{333}$ versus $\log C$ of GA-GEL conjugates for the pyrene excitation spectra, resulting in confirming the CMC obtained from the intersection of two straight lines. The CMC values of GAGEL-1, GA-GEL-2, and GA-GEL-3 were 0.100, 0.074, and $0.056 \mathrm{mg} / \mathrm{mL}$, respectively. Low CMC values demonstrated that these copolymers could be easy to form micelles and keep perfect stability even under highly diluted conditions in vivo. The CMC values of three kinds of copolymers exhibited the trend of gradual decrease with the increase content of GA groups. The reason was that self-assembled activity of the micelles in aqueous media was improved as the mass of hydrophobic GA increased [34].

The core-shell GA-GEL micelles could be formed by self-assembly in aqueous media. As shown in Table 1, the mean diameters of blank micelles were ranged from 119 to $134 \mathrm{~nm}$. And the particle sizes increased as the substitution degrees of GA increased. The result was possibly ascribed to the fact that hydrophobic groups occupied the core space in the micelles [25]. The zeta potentials of GA-GEL-1, GAGEL-2, and GA-GEL-3 micelles were 14.2, 13.4, and $15.1 \mathrm{mV}$, respectively. The mean diameters of DOX-loaded micelles were approximately from 195 to $235 \mathrm{~nm}$. And the particle size of drug-loaded micelles was larger than that of their blank polymeric micelles. It was possibly attributed to the fact that DOX molecules were loaded into the micelles and the inner space of these micelles increased [35]. The zeta potentials of DOX-loaded micelles were ranged from 13.8 to $14.9 \mathrm{mV}$. And the particle sizes of blank and DOX-loaded micelles did not change in aqueous media for $48 \mathrm{~h}$ at room temperature. The loading content (LC) of DOX/GA-GEL-1, DOX/GA-GEL-2, and DOX/GA-GEL-3 micelles were 10.9\%, 12.5\%, and 8.3\%, respectively (Table 1). It was found that DOX/GA-GEL-2 micelles showed the highest LC and encapsulation efficiency (EE) compared with DOX/GA-GEL-1 and DOX/GAGEL-3 micelles. According to the above physicochemical characteristics, DOX/GA-GEL-2 micelles were selected for further evaluation in vitro and in vivo.

As shown in Figure 4, the morphology of GA-GEL-2 and DOX/GA-GEL-2 micelles was approximately spherical observed by transmission electron microscopy (TEM). The particle sizes determined by TEM were smaller than that analyzed by dynamic light scattering (DLS) (Table 1). It was due to the fact that the particles were in dry state determined by TEM, while DLS analysis process was performed in hydrated state $[36,37]$.

3.2. In Vitro Drug Release. DOX release from drug-loaded micelles was investigated in $\mathrm{PBS}$ at $\mathrm{pH} 7.4$, imitating the physiological environment in vivo. As shown in Figure 5, DOX from DOX/GA-GEL-2 micelles released about 39.5\% for $8 \mathrm{~h}$ and $41.7 \%$ for $72 \mathrm{~h}$. DOX release from drug-loaded micelles revealed a biphasic pattern, which was an initial burst release and a following slower and sustained release.

3.3. In Vitro Cellular Uptake. The in vitro cellular uptake of DOX/GA-GEL-2 in HepG2 cells was studied by CLSM. As shown in Figure 6, red fluorescence was emitted from DOX formulations and blue fluorescence stained in nuclei was from Hoechst 33258. The fluorescence signals of $\mathrm{DOX} \cdot \mathrm{HCl}$ were stronger than that of DOX/GA-GEL-2 micelles in $2 \mathrm{~h}$ (Figure 6(a)). As DOX-loaded micelles were further incubated for $6 \mathrm{~h}$, the red fluorescence intensity was obviously improved (Figure 6(b)). Hence, the cellular uptake of DOX/GA-GEL-2 and $\mathrm{DOX} \cdot \mathrm{HCl}$ was time-dependent in HepG2 cells. Notably, 


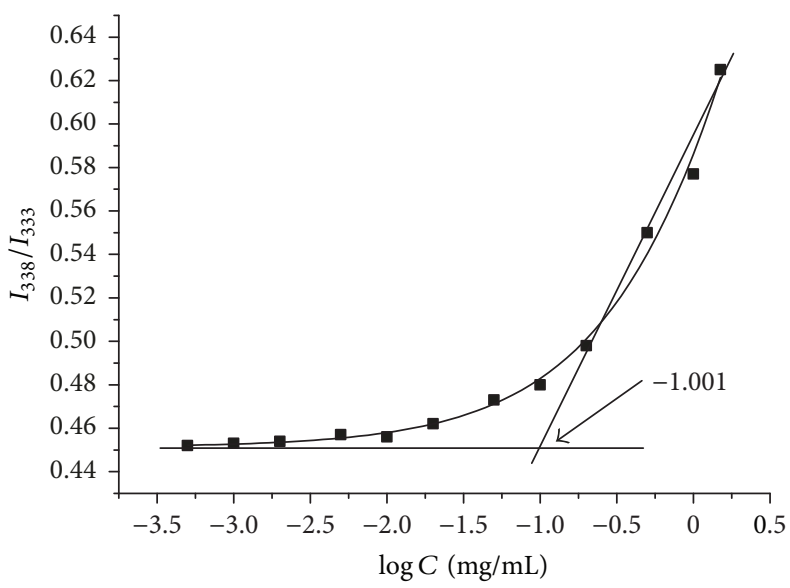

(a)

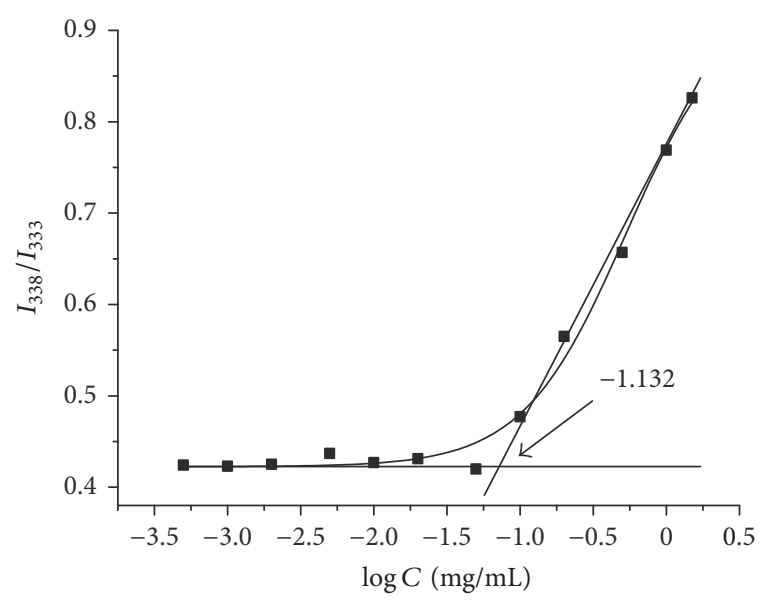

(b)

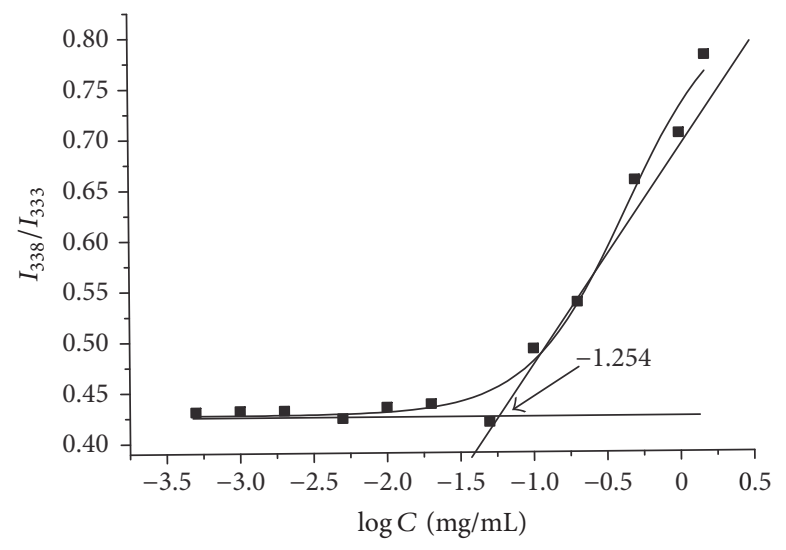

(c)

FIGURE 3: Plot of the intensity ratio $I_{338} / I_{333}$ from pyrene excitation spectra of (a) GA-GEL-1, (b) GA-GEL-2, and (c) GA-GEL-3 as a function of $\log C$.

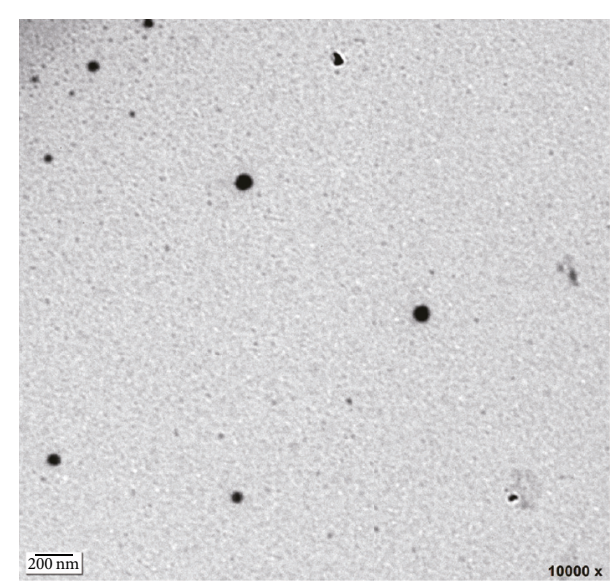

(a)

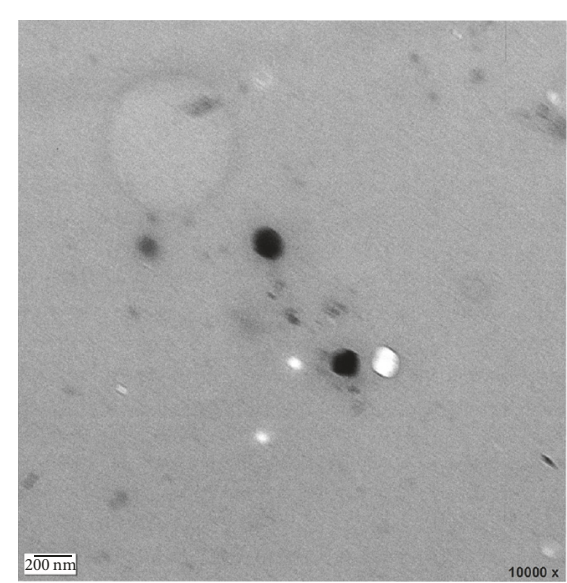

(b)

FIGURE 4: Transmission electron microscope images of (a) GA-GEL-2 and (b) DOX/GA-GEL-2 micelles. 


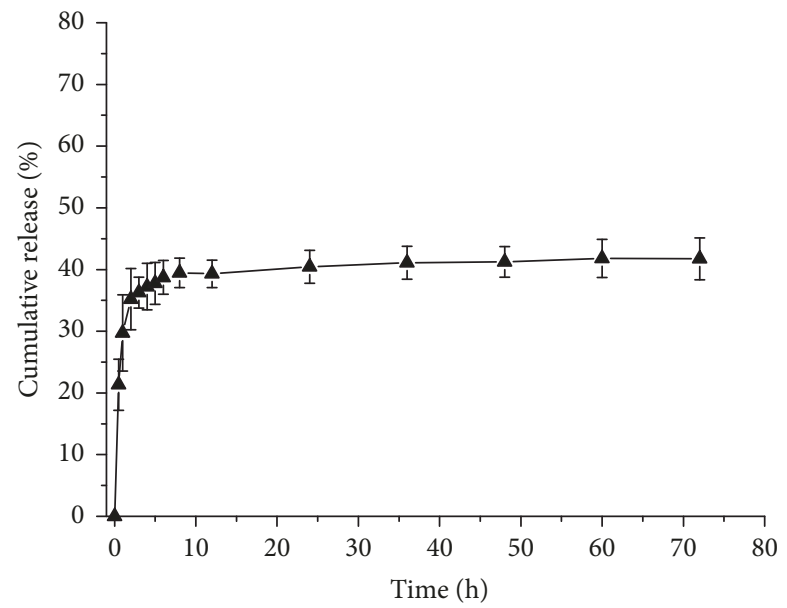

Figure 5: Release profiles of DOX from DOX/GA-GEL-2 micelles in PBS (pH 7.4) at $37^{\circ} \mathrm{C}$ for $72 \mathrm{~h}$.

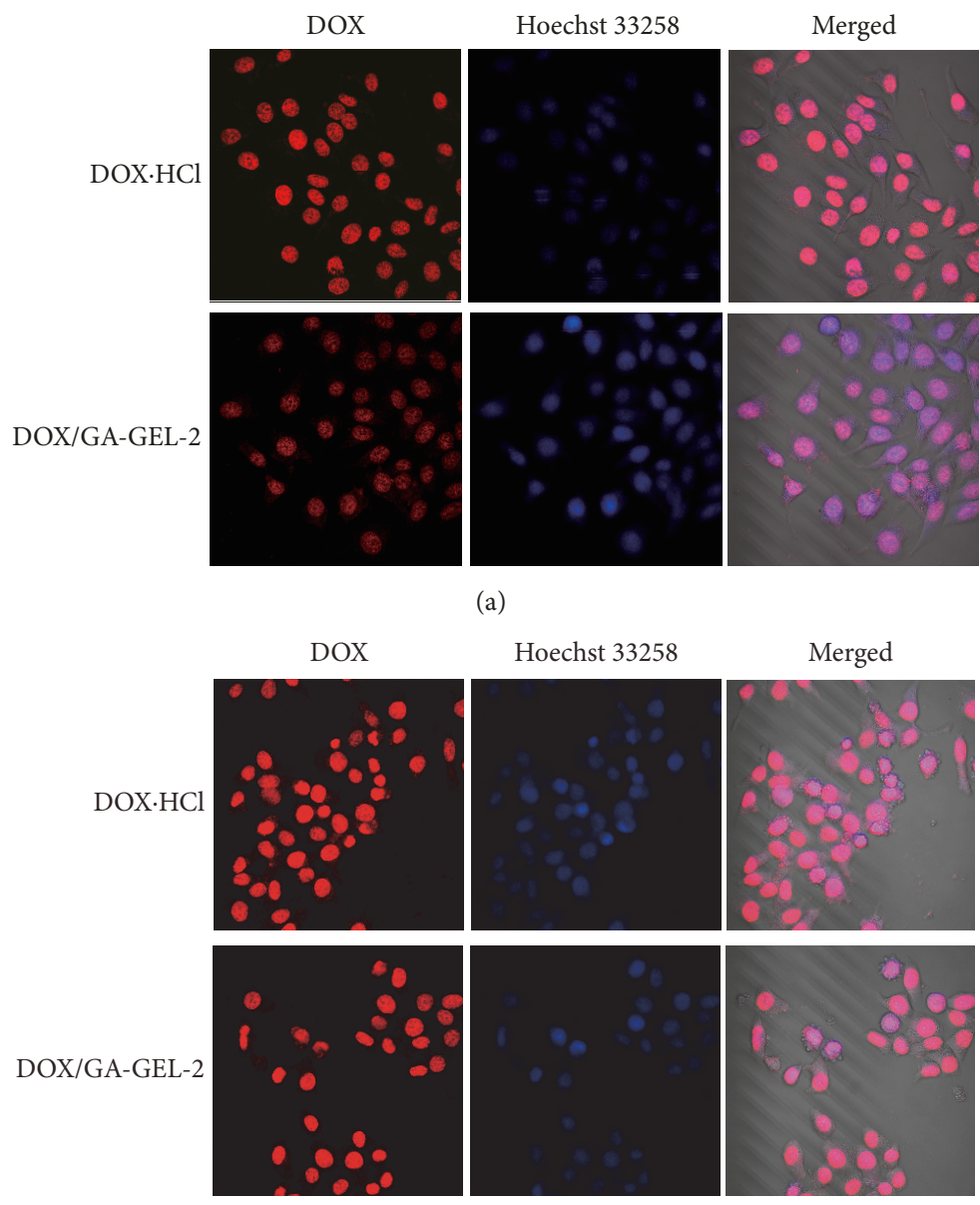

(b)

Figure 6: CLSM images of HepG2 cells after incubation with DOX.HCl and DOX/GA-GEL-2 micelles for (a) $2 \mathrm{~h}$ and (b) $6 \mathrm{~h}$. The blue fluorescence was from nucleus staining by Hoechst 33258. The scale bars were $20 \mu \mathrm{m}$ in all the images. 

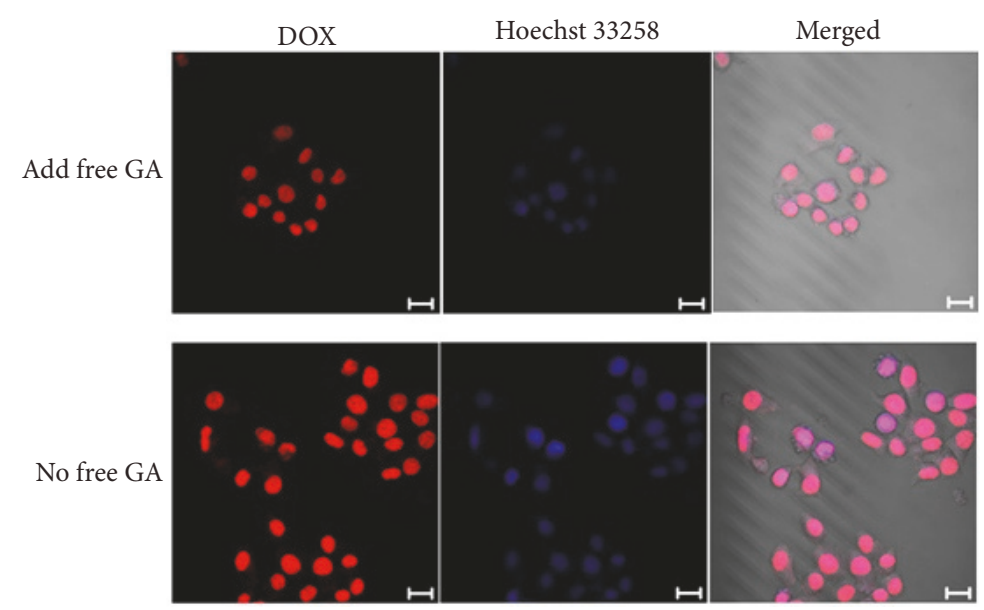

FIGURE 7: CLSM images of HepG2 cells pretreated with free GA or without GA after incubation with DOX/GA-GEL-2 micelles for 6 h.

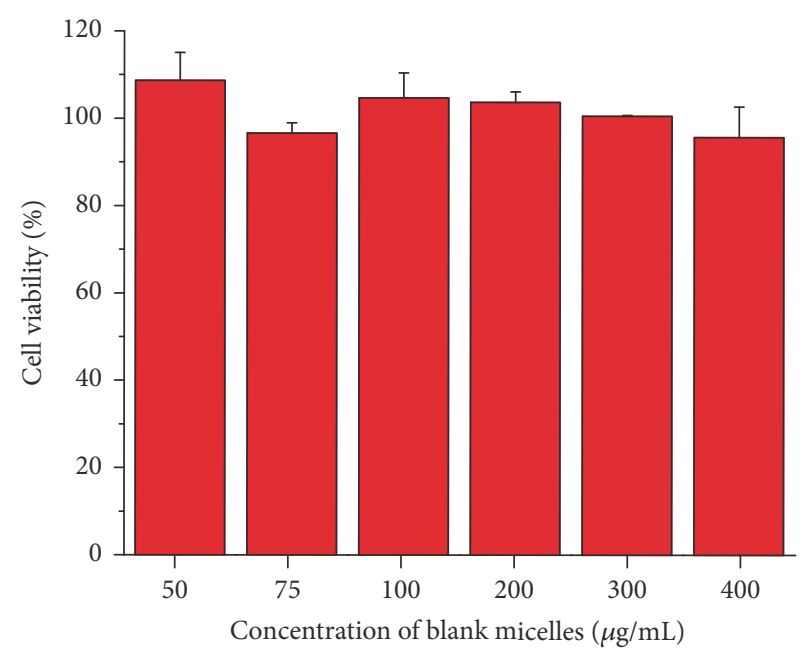

(a)

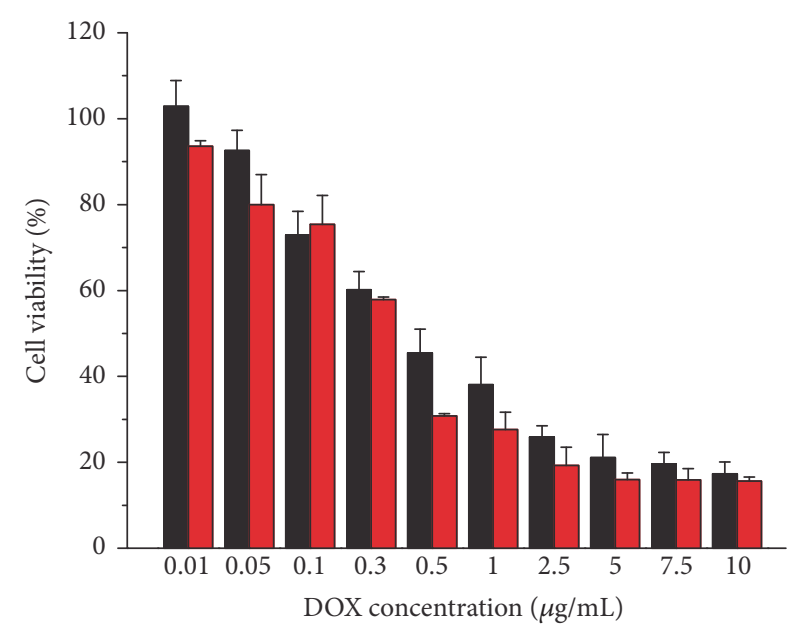

DOX $\mathrm{HCl}$

DOX/GA-GEL-2

(b)

FIGURE 8: The in vitro cytotoxicity of (a) GA-GEL-2 micelles and (b) DOX.HCl and DOX/GA-GEL-2 micelles against HepG2 cells after $48 \mathrm{~h}$ incubation.

the fluorescence intensity of DOX/GA-GEL-2 micelles was equivalent to $\mathrm{DOX} \cdot \mathrm{HCl}$ in $6 \mathrm{~h}$ (Figure $6(\mathrm{~b})$ ). As previously reported, drug-loaded micelles were transported into the cells by an energy-dependent endocytosis way, and DOX. $\mathrm{HCl}$ could quickly enter the cells by a passive diffusion manner [38]. The drug-loaded micelles were entered into tumor cells in a slow way. Increasing drugs were released from DOX-loaded micelles and distributed within the cells in $6 \mathrm{~h}$. Further, DOX fluorescence from DOX-loaded micelles was mainly distributed in nuclei, which was similar to DOX.HCl. Therefore, DOX-loaded micelles had the advantages of desirable particle size and cellular uptake, which provided the potential for targeting HCC.

It was known that GA could specially bind with GA receptor in hepatoma cells [39]. Then further investigation was adopted to study the targeting ability of drug-loaded GAGEL micelles. HepG2 cells were preincubated $2 \mathrm{~h}$ with free GA, followed by treatment of DOX/GA-GEL-2 micelles As shown in Figure 7, the fluorescence intensity of DOX/GAGEL-2 micelles with adding free GA in HepG2 cells was lower than that of the micelles without free GA. The result was ascribed to the fact that GA receptors on the surface of HepG2 cells were occupied by free GA, leading to the decrease of transportation of DOX-loaded GA-GEL micelles into the cells.

3.4. In Vitro Cytotoxicity. In vitro cytotoxicity of blank or DOX-loaded micelles was studied by MTT assay against HepG2 cells. As shown in Figure 8(a), blank GA-GEL2 micelles had no cytotoxicity in the concentration of 

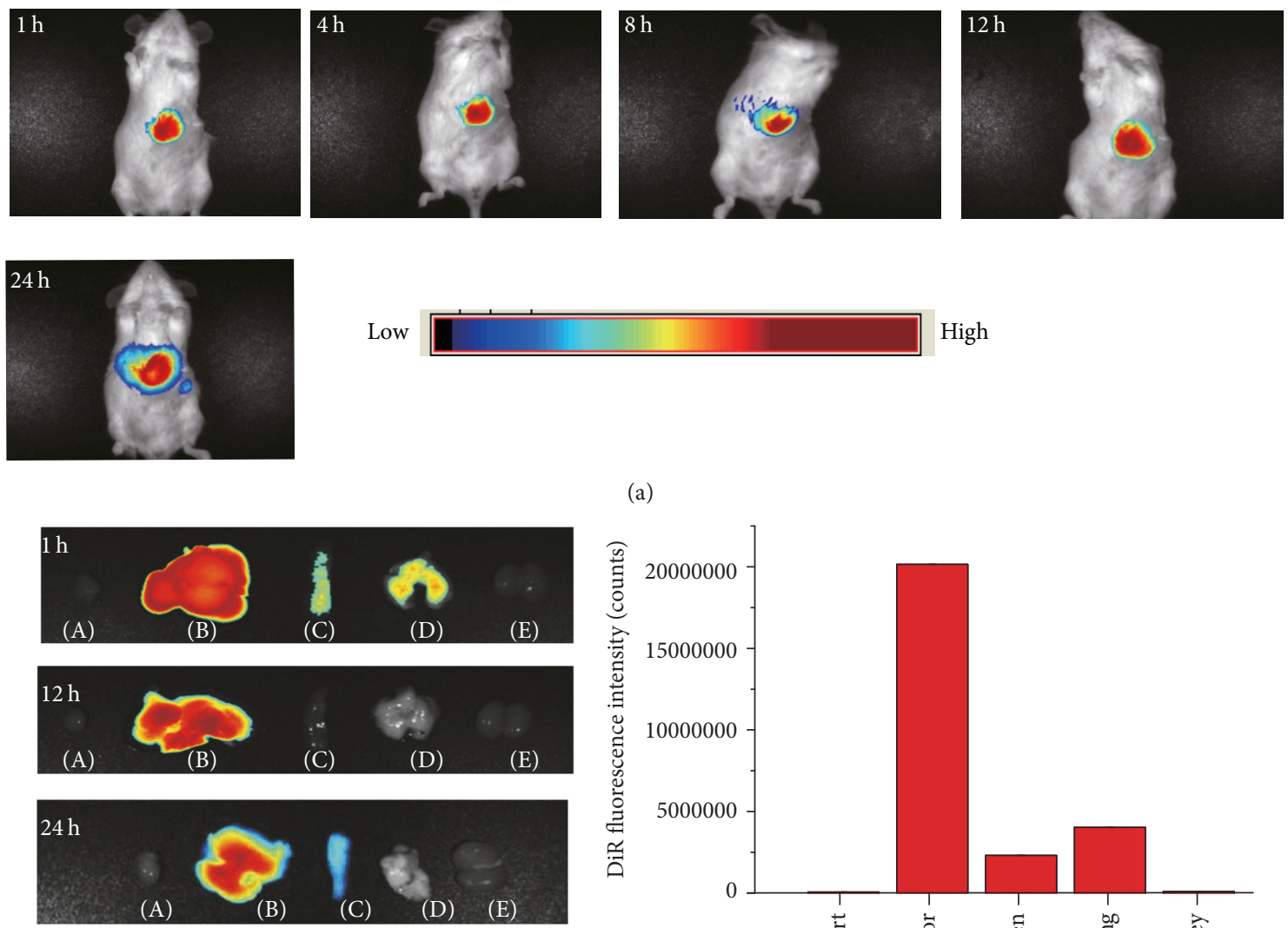

(a)

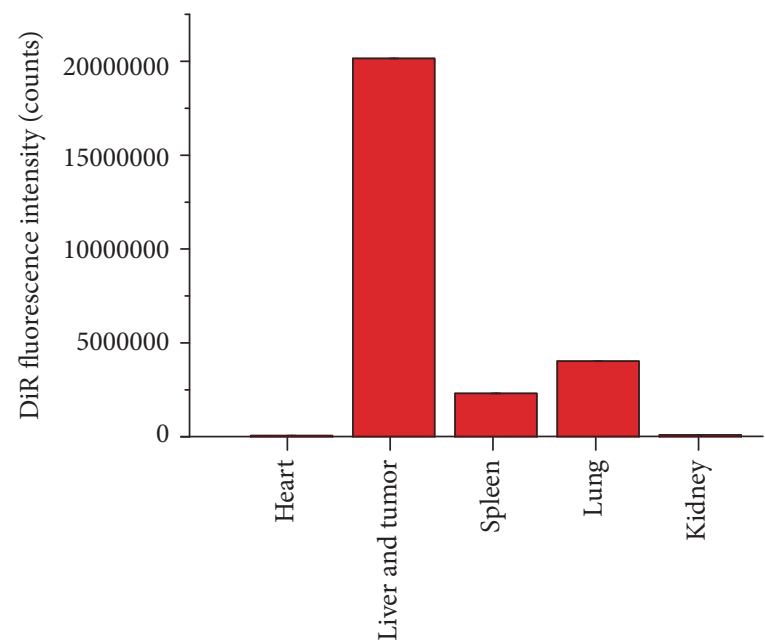

(b)

(c)

FIGURE 9: Fluorescence imaging of mice administrated with DiR-labeled GA-GEL-2 micelles. (a) Time-dependent in vivo images of mice bearing HCC tumors after i.v. injection. (b) Ex vivo NIR fluorescence image of dissected organs after 1, 12, and $24 \mathrm{~h}$ after injection. (A), heart; (B), liver and tumor; (C), spleen; (D), lung; and (E), kidney. (c) Fluorescence intensities of orthotopic tumors and dissected organs after $1 \mathrm{~h}$ after injection.

$50-400 \mu \mathrm{g} / \mathrm{mL}$. It would eliminate the possibility that GA was responsible for the cytotoxicity. In Figure $8(\mathrm{~b})$, the cytotoxicity of DOX formulations exhibited concentration dependence. Additionally, the $\mathrm{IC}_{50}$ values of DOX/GA-GEL2 and DOX $\mathrm{HCl}$ micelles were 0.33 and $0.66 \mu \mathrm{g} / \mathrm{mL}$, respectively. DOX/GA-GEL-2 micelles were 2 -fold stronger $(P<$ $0.05)$ than DOX $\cdot \mathrm{HCl}$. The result showed that DOX-loaded micelles had strong targeting potential for hepatocellular carcinoma in vitro. It was ascribed to the fact that DOXloaded micelles could be effectively internalized by HepG2 cells, and GEL-based micelles were degraded by MMP- 2 in the cells, leading to drug fast release. It was consistent with the result of CLSM observations.

3.5. In Vivo Imaging Analysis. The orthotopic H22 tumorbearing mice were applied to study in vivo imaging analysis. To assess the liver-targeting activity of drug-loaded micelles, the biodistribution of DiR-loaded GA-GEL-2 micelles was monitored by noninvasive near infrared optical imaging technique. GA-GEL-2 micelles represented a remarkable fluorescence signals in liver and orthotopic tumor sites $1 \mathrm{~h}$ after injection (Figure 9). These fluorescence signals were still observed in liver and orthotopic tumor at $24 \mathrm{~h}$. Therefore, GA-GEL-2 micelles possessed targeting HCC, and the strong fluorescence signals could keep a long time in liver and orthotopic tumor. As presented in Figure 9(b), the fluorescence signals of liver and orthotopic tumor were significantly stronger than other organs at predetermined time. Moreover, the statistical data of biodistribution showed that the fluorescence intensity of heart and kidney was negligible at $1 \mathrm{~h}$ (Figure 9(c)). These results infer that GAGEL-2 micelles have remarkable targetability and possess the potential to reduce side effects of cardiotoxicity and nephrotoxicity caused by DOX [33].

3.6. In Vivo Antitumor Efficacy and Safety Evaluation. The orthotopic H22 tumor-bearing mice were used to perform in vivo antitumor efficacy and safety evaluation. The H22 


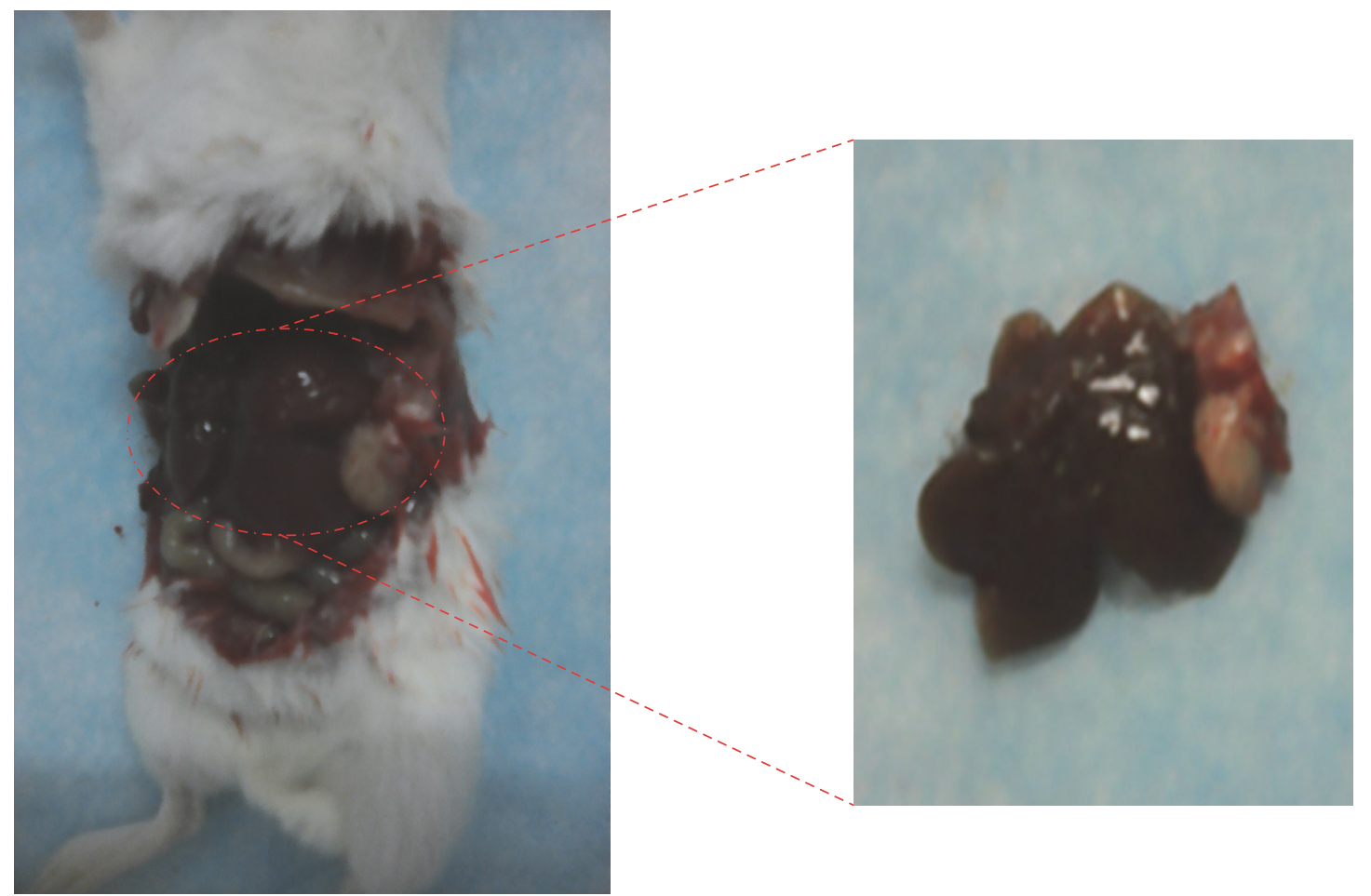

(a)

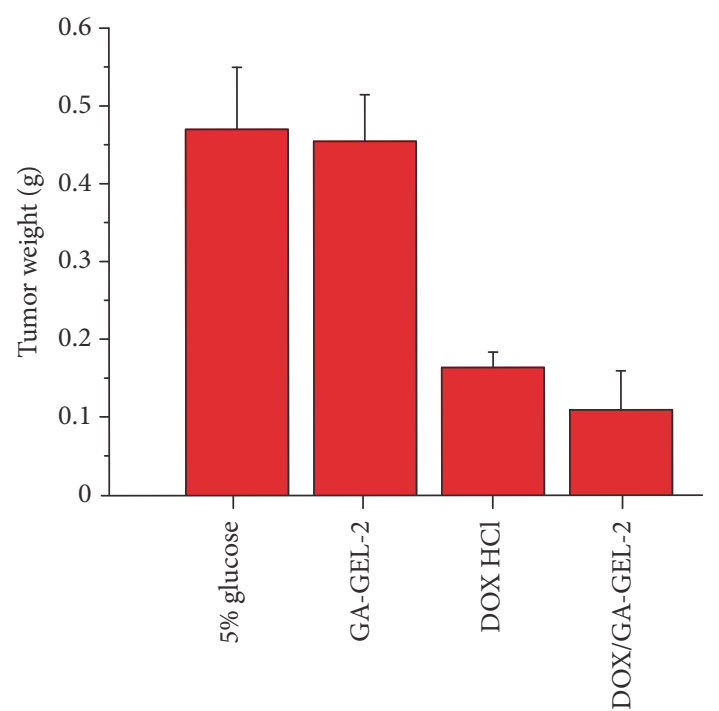

(b)

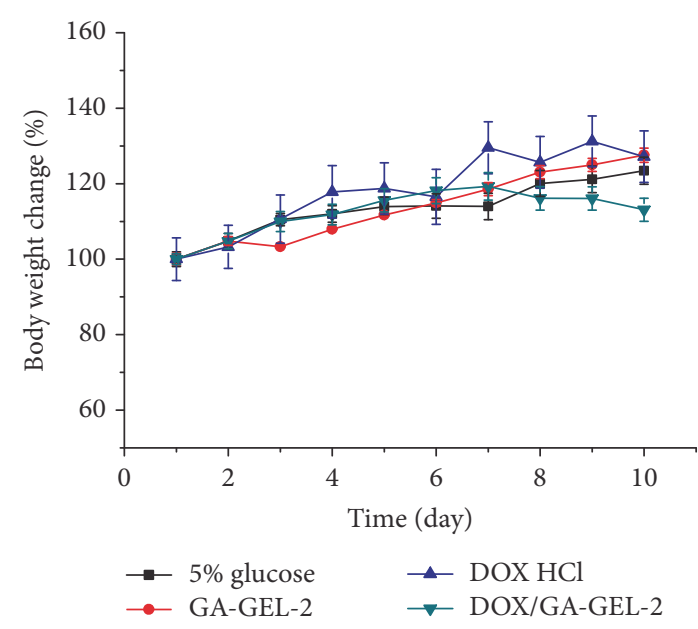

(c)

FIGURE 10: In vivo antitumor activity of 5\% glucose, DOX.HCl, GA-GEL-2, and DOX/GA-GEL-2 micelles in H22-bearing mice: (a) H22 orthotopic xenograft tumor model, (b) mice tumor weight on 10th day, and (c) body weight change within 10 day.

orthotopic xenograft model was established in Figure 10(a). The tumor weight of DOX/GA-GEL-2 micelles groups was significantly less $(P<0.05)$ than $5 \%$ glucose and GAGEL-2 micelles groups and was less than DOX $\cdot \mathrm{HCl}$ group (Figure 10(b)). The tumor inhibitory rates (TIR) of DOX/GAGEL-2 micelles and DOX.HCl were $76.9 \%$ and $65.3 \%$, respectively. These results demonstrated that DOX/GA-GEL2 micelles exhibited better inhibitory potency in $\mathrm{H} 22$-bearing orthotopic tumor. The passive and positive targeting effects could be the main reasons for the significant suppression of tumor growth in DOX-encapsulated micelles [40]. In addition, MMP-2 was overexpressed in the tumor sites [41]. It was known that gelatin could be degraded by MMP2. Then, DOX would be rapidly released from DOX/GAGEL-2 micelles. Similar phenomenon was described by other researchers [10]. As shown in Figure 10(c), after being treated with DOX.HCl and DOX/GA-GEL-2 micelles, the mice weight change increased slightly compared with the 


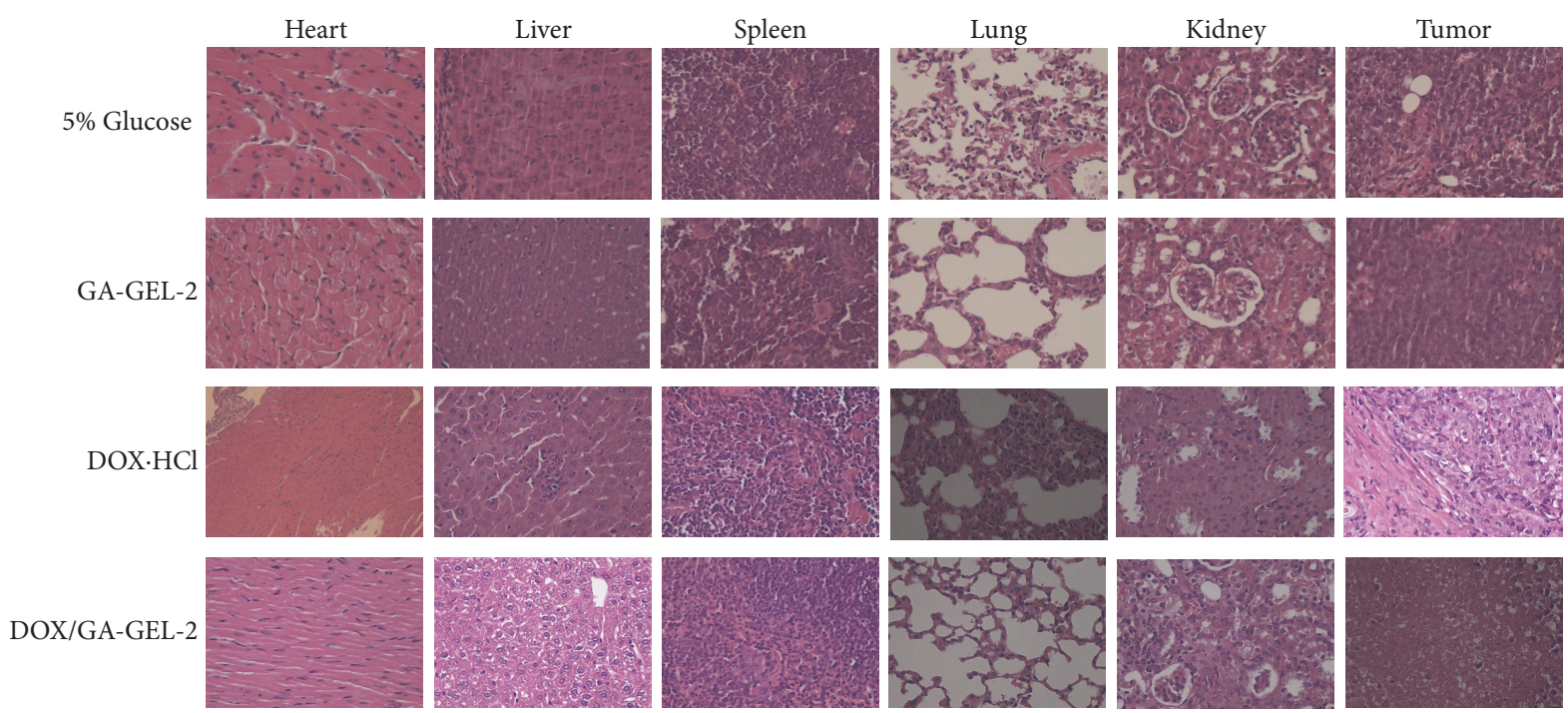

FIGURE 11: Pathological images of tumors and organs with H\&E staining in H22 orthotopic xenograft mice after treatment on 10th day. Light microscopy images were obtained under Leica microscope.

initial weight. According to the observation throughout the experiment, two mice in DOX.HCl group died on days 8 and 9 , and the status of these mice was bad. The phenomenon was not seen in the other groups. These results demonstrated that drug-loaded micelles could decrease toxicity caused by DOX.

The toxicity is always a key concern for nanoparticulate system used in biomedicine. To further evaluate the safety and effectiveness of drug delivery system, the toxicity and tumor apoptosis of DOX/GA-GEL-2 micelles were studied by histochemistry analysis. As shown in Figure 11, no noticeable signals of organ damages in DOX/GA-GEL-2 group were observed from heart, liver, spleen, lung, and kidney. The result was consistent with the result of in vivo imaging analysis. However, DOX. $\mathrm{HCl}$ group exhibited organ damages including cardiotoxicity. Moreover, there was more obvious tumor apoptosis in DOX/GA-GEL-2 micelles than that in DOX.HCl. Further, there was no significant tumor apoptosis in 5\% glucose and GA-GEL-2 groups. These results suggested that DOX/GA-GEL-2 micelles could be safe and effective drug delivery vehicles for tumor chemotherapy.

\section{Conclusions}

GA-GEL conjugates with different substitution degrees have been synthesized and used as targeting hepatocellular carcinoma vehicles for DOX delivery. GA-GEL copolymers had low CMC values. And DOX-loaded micelles showed high drug-loading content. DOX-loaded micelles could effectively be transported and accumulated in hepatoma cells. DOX/GA-GEL-2 micelles demonstrated higher cellular uptake and cytotoxicity than DOX. $\mathrm{HCl}$ in $\mathrm{HepG} 2$ cells. The in vivo imaging analysis showed that DiR-labeled GAGEL-2 micelles had liver targetability. Furthermore, the safety evaluation studies indicated that GA-GEL-2 micelles had no hepatic or systemic toxicity. And DOX/GA-GEL-2 micelles exhibited stronger tumor inhibition than DOX $\mathrm{HCl}$ in orthotopic $\mathrm{H} 22$ tumor-bearing mice. Taken together, GAGEL micelles could be a potential targeting drug carrier for HCC therapy.

\section{Conflicts of Interest}

The authors report no conflicts of interest.

\section{Acknowledgments}

This work was financially supported by National Natural Science Foundation of China (81360484), Natural Science Foundation of Jiangxi Province (20151BAB205081), and Scientific Research Fund of Jiangxi Health Department (20157104).

\section{References}

[1] J. L. Petrick, M. Braunlin, M. Laversanne, P. C. Valery, F. Bray, and K. A. McGlynn, "International trends in liver cancer incidence, overall and by histologic subtype, 1978-2007," International Journal of Cancer, vol. 139, no. 7, pp. 1534-1545, 2016.

[2] P. Pathak, V. Dhawan, A. Magarkar et al., "Design of cholesterol arabinogalactan anchored liposomes for asialoglycoprotein receptor mediated targeting to hepatocellular carcinoma: in silico modeling, in vitro and in vivo evaluation," International Journal of Pharmaceutics, vol. 509, no. 1-2, pp. 149-158, 2016.

[3] D. P. Otto, A. Otto, and M. M. De Villiers, "Differences in physicochemical properties to consider in the design, evaluation and choice between microparticles and nanoparticles for drug delivery," Expert Opinion on Drug Delivery, vol. 12, no. 5, pp. 763-777, 2015.

[4] L. Dai, Y. Yu, Z. Luo et al., "Photosensitizer enhanced disassembly of amphiphilic micelle for ROS-response targeted tumor therapy in vivo," Biomaterials, vol. 104, pp. 1-17, 2016.

[5] J. Yu, Y. Zhou, W. Chen et al., "Preparation, characterization and evaluation of $\alpha$-tocopherol succinate-modified dextran micelles 
as potential drug carriers," Materials , vol. 8, no. 10, pp. 66856696, 2015.

[6] H.-L. Ma, X.-R. Qi, Y. Maitani, and T. Nagai, "Preparation and characterization of superparamagnetic iron oxide nanoparticles stabilized by alginate," International Journal of Pharmaceutics, vol. 333, no. 1-2, pp. 177-186, 2007.

[7] P. R. Sarika, N. R. James, P. R. Anil kumar, and D. K. Raj, "Preparation, characterization and biological evaluation of curcumin loaded alginate aldehyde-gelatin nanogels," Materials Science and Engineering C: Materials for Biological Applications, vol. 68, pp. 251-257, 2016.

[8] C. Zhang, W. Wang, T. Liu et al., "Doxorubicin-loaded glycyrrhetinic acid-modified alginate nanoparticles for liver tumor chemotherapy," Biomaterials, vol. 33, no. 7, pp. 2187-2196, 2012.

[9] H. Liu, S. Wu, J. Yu et al., "Reduction-sensitive micelles selfassembled from amphiphilic chondroitin sulfate A-deoxycholic acid conjugate for triggered release of doxorubicin," Materials Science and Engineering C: Materials for Biological Applications, vol. 75, pp. 55-63, 2017.

[10] C. Wong, T. Stylianopoulos, J. Cui et al., "Multistage nanoparticle delivery system for deep penetration into tumor tissue," Proceedings of the National Acadamy of Sciences of the United States of America, vol. 108, no. 6, pp. 2426-2431, 2011.

[11] A. K. Bajpai and J. Choubey, "Design of gelatin nanoparticles as swelling controlled delivery system for chloroquine phosphate," Journal of Materials Science: Materials in Medicine, vol. 17, no. 4, pp. 345-358, 2006.

[12] H. Chen, X. Xing, H. Tan et al., "Covalently antibacterial alginate-chitosan hydrogel dressing integrated gelatin microspheres containing tetracycline hydrochloride for wound healing," Materials Science and Engineering C: Materials for Biological Applications, vol. 70, no. 2, pp. 287-295, 2017.

[13] M. Negishi, A. Irie, N. Nagata, and A. Ichikawa, "Specific binding of glycyrrhetinic acid to the rat liver membrane," $B B A$ - Biomembranes, vol. 1066, no. 1, pp. 77-82, 1991.

[14] I. B. Bwatanglang, F. Mohammad, N. A. Yusof et al., "In vivo tumor targeting and anti-tumor effects of 5-fluororacil loaded, folic acid targeted quantum dot system," Journal of Colloid and Interface Science, vol. 480, pp. 146-158, 2016.

[15] W. Chen, F. Meng, R. Cheng, C. Deng, J. Feijen, and Z. Zhong, "Biodegradable glycopolymer-b-poly( $\varepsilon$-caprolactone) block copolymer micelles: versatile construction, tailored lactose functionality, and hepatoma-targeted drug delivery," Journal of Materials Chemistry B, vol. 3, no. 11, pp. 2308-2317, 2015.

[16] J. Li, H. Xu, X. Ke, and J. Tian, "The anti-tumor performance of docetaxel liposomes surface-modified with glycyrrhetinic acid," Journal of Drug Targeting, vol. 20, no. 5, pp. 467-473, 2012.

[17] F. Chen, J. Zhang, Y. He, X. Fang, Y. Wang, and M. Chen, "Glycyrrhetinic acid-decorated and reduction-sensitive micelles to enhance the bioavailability and anti-hepatocellular carcinoma efficacy of tanshinone IIA," Biomaterials Science, vol. 4, no. 1, pp. 167-182, 2016.

[18] L. Zhang, J. Yao, J. Zhou, T. Wang, and Q. Zhang, "Glycyrrhetinic acid-graft-hyaluronic acid conjugate as a carrier for synergistic targeted delivery of antitumor drugs," International Journal of Pharmaceutics, vol. 441, no. 1-2, pp. 654-664, 2013.

[19] J. Chen, H. Jiang, Y. Wu, Y. Li, and Y. Gao, "A novel glycyrrhetinic acid-modified oxaliplatin liposome for livertargeting and in vitro/vivo evaluation," Drug Design, Development and Therapy, vol. 9, pp. 2265-2275, 2015.

[20] M. Cheng, X. Gao, Y. Wang et al., "Synthesis of liver-targeting dual-ligand modified GCGA/5-FU nanoparticles and their characteristics in vitro and in vivo," International Journal of Nanomedicine, vol. 8, pp. 4265-4276, 2013.

[21] Q. Tian, X.-H. Wang, W. Wang, C.-N. Zhang, P. Wang, and Z. Yuan, "Self-assembly and liver targeting of sulfated chitosan nanoparticles functionalized with glycyrrhetinic acid," Nanomedicine: Nanotechnology, Biology and Medicine, vol. 8, no. 6, pp. 870-879, 2012.

[22] Y. Cong, B. Shi, Y. Lu, S. Wen, R. Chung, and D. Jin, “Onestep Conjugation of Glycyrrhetinic Acid to Cationic Polymers for High-performance Gene Delivery to Cultured Liver Cell," Scientific Reports, vol. 6, Article ID 21891, 2016.

[23] J.-M. Yu, W.-D. Li, L. Lu et al., "Preparation and characterization of galactosylated glycol chitosan micelles and its potential use for hepatoma-targeting delivery of doxorubicin," Journal of Materials Science: Materials in Medicine, vol. 25, no. 3, pp. 691701, 2014.

[24] M. S. Sudheesh, S. P. Vyas, and D. V. Kohli, "Nanoparticlebased immunopotentiation via tetanus toxoid-loaded gelatin and aminated gelatin nanoparticles," Drug Delivery, vol. 18, no. 5, pp. 320-330, 2011.

[25] W.-M. Li, D.-M. Liu, and S.-Y. Chen, "Amphiphilicallymodified gelatin nanoparticles: Self-assembly behavior, controlled biodegradability, and rapid cellular uptake for intracellular drug delivery," Journal of Materials Chemistry, vol. 21, no. 33, pp. 12381-12388, 2011.

[26] L. Yan, W. Wu, W. Zhao et al., "Reduction-sensitive core-crosslinked mPEG-poly(ester-carbonate) micelles for glutathionetriggered intracellular drug release," Polymer Chemistry, vol. 3, no. 9, pp. 2403-2412, 2012.

[27] C.-G. Liu, K. G. H. Desai, X.-G. Chen, and H.-J. Park, "Linolenic acid-modified chitosan for formation of selfassembled nanoparticles," Journal of Agricultural and Food Chemistry, vol. 53, no. 2, pp. 437-441, 2005.

[28] Q.-L. Zhu, Y. Zhou, M. Guan et al., "Low-density lipoproteincoupled N-succinyl chitosan nanoparticles co-delivering siRNA and doxorubicin for hepatocyte-targeted therapy," Biomaterials, vol. 35, no. 22, pp. 5965-5976, 2014.

[29] S. Chen, Q. Lei, S.-Y. Li et al., "Fabrication of dual responsive co-delivery system based on three-armed peptides for tumor therapy," Biomaterials, vol. 92, pp. 25-35, 2016.

[30] F. Zhu, Y. Jiang, F. Luo, and P. Li, "Effectiveness of localized ultrasound-targeted microbubble destruction with doxorubicin liposomes in H22 mouse hepatocellular carcinoma model," Journal of Drug Targeting, vol. 23, no. 4, pp. 323-334, 2015.

[31] X. Wang, X. Gu, H. Wang, Y. Sun, H. Wu, and S. Mao, "Synthesis, characterization and liver targeting evaluation of self-assembled hyaluronic acid nanoparticles functionalized with glycyrrhetinic acid," European Journal of Pharmaceutical Sciences, vol. 96, pp. 255-262, 2017.

[32] X. Jiang, H. Xin, J. Gu et al., "Solid tumor penetration by integrin-mediated pegylated poly(trimethylene carbonate) nanoparticles loaded with paclitaxel," Biomaterials, vol. 34, no. 6, pp. 1739-1746, 2013.

[33] L. Shi, C. Tang, and C. Yin, "Glycyrrhizin-modified O-carboxymethyl chitosan nanoparticles as drug vehicles targeting hepatocellular carcinoma," Biomaterials, vol. 33, no. 30, pp. 7594-7604, 2012.

[34] V. A. Burilov, D. A. Mironova, R. R. Ibragimova, R. I. Nugmanov, S. E. Solovieva, and I. S. Antipin, "Detection of sulfate surface-active substances via fluorescent response using new amphiphilic thiacalix[4]arenes bearing cationic headgroups 
with Eosin Y dye," Colloids and Surfaces A: Physicochemical and Engineering Aspects, vol. 515, pp. 41-49, 2017.

[35] Y. Zhou, J. Yu, X. Feng et al., "Reduction-responsive corecrosslinked micelles based on a glycol chitosan-lipoic acid conjugate for triggered release of doxorubicin," RSC Advances, vol. 6, no. 37, pp. 31391-31400, 2016.

[36] B. Khorsand, G. Lapointe, C. Brett, and J. K. Oh, "Intracellular drug delivery nanocarriers of glutathione-responsive degradable block copolymers having pendant disulfide linkages," Biomacromolecules, vol. 14, no. 6, pp. 2103-2111, 2013.

[37] C.-Y. Yu, L.-H. Jia, B.-C. Yin, X.-Z. Zhang, S.-X. Cheng, and R.-X. Zhuo, "Fabrication of nanospheres and vesicles as drug carriers by self-assembly of alginate," The Journal of Physical Chemistry C, vol. 112, no. 43, pp. 16774-16778, 2008.

[38] J. Yu, X. Xie, M. Zheng et al., "Fabrication and characterization of nuclear localization signal-conjugated glycol chitosan micelles for improving the nuclear delivery of doxorubicin," International Journal of Nanomedicine, vol. 7, pp. 5079-5090, 2012.

[39] H. Guo, Q. Lai, W. Wang et al., "Functional alginate nanoparticles for efficient intracellular release of doxorubicin and hepatoma carcinoma cell targeting therapy," International Journal of Pharmaceutics, vol. 451, no. 1-2, pp. 1-11, 2013.

[40] Q. Chen, H. Ding, J. Zhou et al., "Novel glycyrrhetinic acid conjugated $\mathrm{pH}$-sensitive liposomes for the delivery of doxorubicin and its antitumor activities," RSC Advances, vol. 6, no. 22, pp. 17782-17791, 2016.

[41] Y. Zhu, W. Meng, H. Gao, and N. Hanagata, "Hollow mesoporous silica/poly(l-lysine) particles for codelivery of drug and gene with enzyme-triggered release property," The Journal of Physical Chemistry C, vol. 115, no. 28, pp. 13630-13636, 2011. 


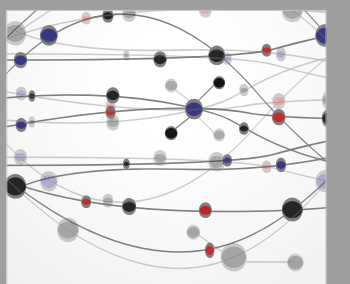

The Scientific World Journal
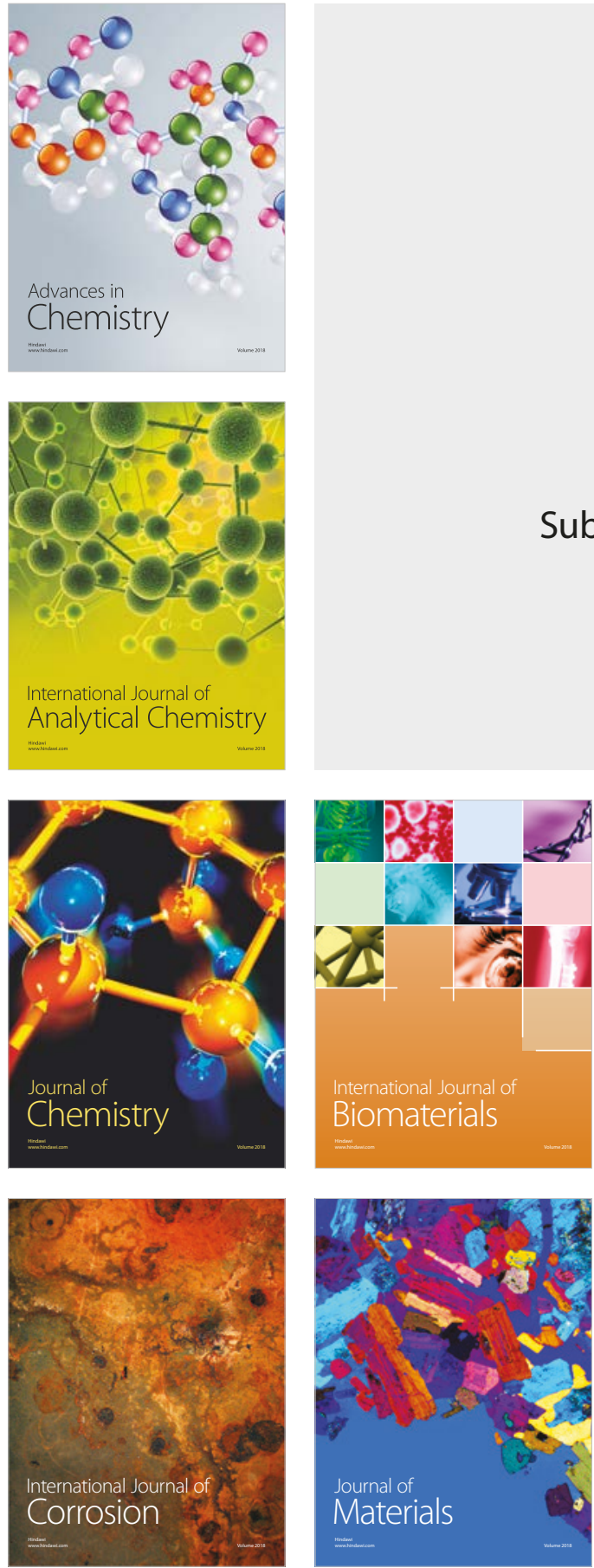

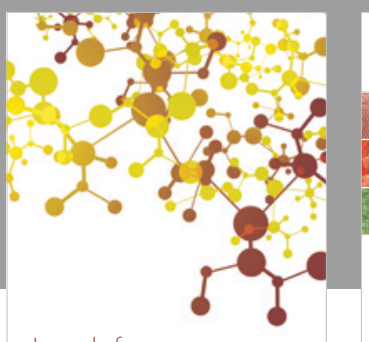

Journal of

Applied Chemistry
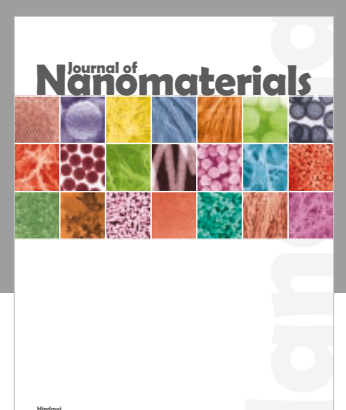

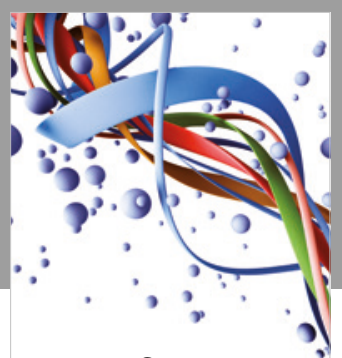

Scientifica

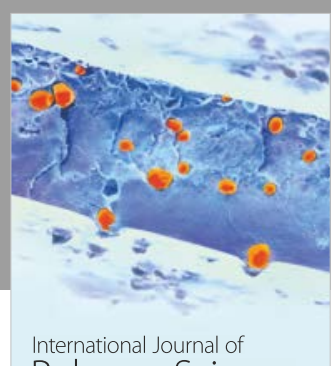

Polymer Science

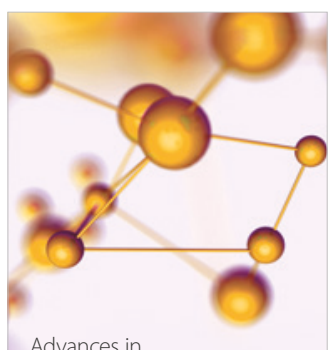

Physical Chemistry
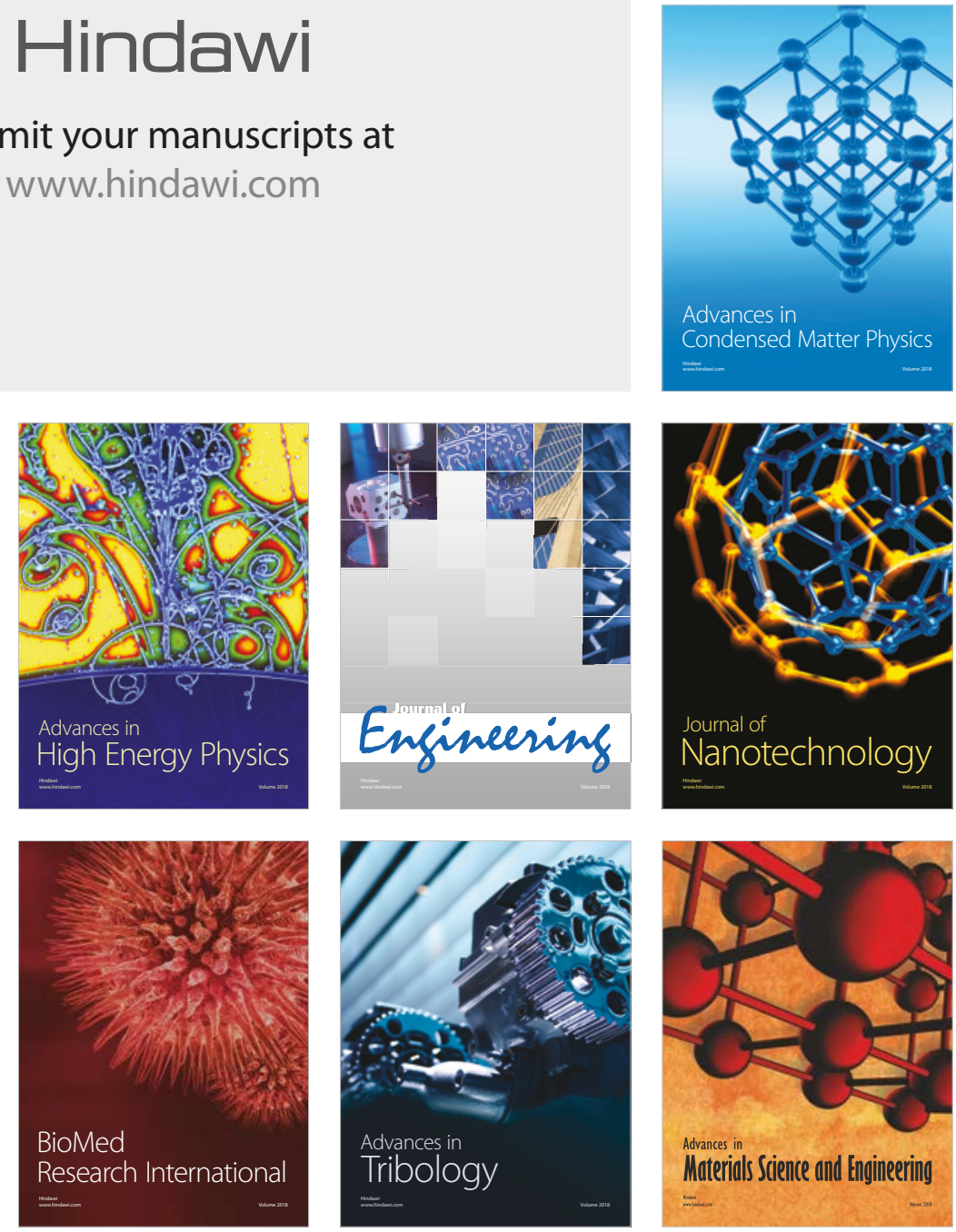